\title{
Updates on Prevention of Hemorrhagic and Lacunar Strokes
}

\author{
Hsin-Hsi Tsai, ${ }^{\mathrm{a}, \mathrm{b}}$ Jong S. Kim, ${ }^{\mathrm{c}}$ Eric Jouvent, ${ }^{\mathrm{d}}$ M. Edip Gurol ${ }^{\mathrm{e}}$ \\ ${ }^{a}$ Department of Neurology, National Taiwan University Hospital, Taipei, Taiwan \\ 'Department of Neurology, National Taiwan University Hospital Bei-Hu Branch, Taipei, Taiwan \\ 'Department of Neurology, Asan Medical Center, University of Ulsan College of Medicine, Seoul, Korea \\ ${ }^{\mathrm{d} D e p a r t m e n t}$ of Neurology, University Paris Diderot, Paris, France \\ 'Department of Neurology, Massachusetts General Hospital, Harvard Medical School, Boston, MA, USA
}

Intracerebral hemorrhage (ICH) and lacunar infarction (LI) are the major acute clinical manifestations of cerebral small vessel diseases (cSVDs). Hypertensive small vessel disease, cerebral amyloid angiopathy, and hereditary causes, such as Cerebral Autosomal Dominant Arteriopathy with Subcortical Infarcts and Leukoencephalopathy (CADASIL), constitute the three common CSVD categories. Diagnosing the underlying vascular pathology in these patients is important because the risk and types of recurrent strokes show significant differences. Recent advances in our understanding of the CSVD-related radiological markers have improved our ability to stratify ICH risk in individual patients, which helps guide antithrombotic decisions. There are general goodpractice measures for stroke prevention in patients with CSVD, such as optimal blood pressure and glycemic control, while individualized measures tailored for particular patients are often needed. Antithrombotic combinations and anticoagulants should be avoided in CSVD treatment, as they increase the risk of potentially fatal ICH without necessarily lowering LI risk in these patients. Even when indicated for a concurrent pathology, such as nonvalvular atrial fibrillation, nonpharmacological approaches should be considered in the presence of CSVD. More data are emerging regarding the presentation, clinical course, and diagnostic markers of hereditary CSVD, allowing accurate diagnosis, and therefore, guiding management of symptomatic patients. When suspicion for asymptomatic hereditary CSVD exists, the pros and cons of prescribing genetic testing should be discussed in detail in the absence of any curative treatment. Recent data regarding diagnosis, risk stratification, and specific preventive approaches for both sporadic and hereditary cSVDs are discussed in this review article.
Correspondence: M. Edip Gurol Department of Neurology, Massachusetts General Hospital, Hemorrhagic Stroke Research Program, 175 Cambridge Street, Suite 300, Boston, MA 02114, USA

Tel: +1-617-275-2375

Fax: +1-506-700-2420

E-mail: edip@mail.harvard.edu

Received: March 13, 2018

Revised: May 16, 2018

Accepted: May 23, 2018

Keywords Stroke, lacunar; Ischemic stroke; Cerebral hemorrhage; Small vessel disease

\section{Introduction}

Cerebral small vessel diseases (cSVDs)-pathologies in small superficial and deep perforating arteries and arterioles of the brain-result in approximately one-fourth of all acute ischemic strokes, mainly in the form of lacunar infarction (LI). The Trial of Org 10172 in Acute Stroke Treatment (TOAST) criteria defines the term $\mathrm{LI}$, in relation to $\mathrm{CSVD}$, as the combination of (1) presentation with one of the traditional clinical lacunar syndromes, (2) normal computed tomography/magnetic resonance 
imaging (MRI) results or demonstration of a relevant brain stem or subcortical lesion with a diameter $<1.5 \mathrm{~cm}$, and (3) exclusion of an alternate etiology, such as cardiac sources of embolism or large vessel disease.'

Intracerebral hemorrhages (ICH) also constitute a major manifestation of CSVD. ${ }^{2}$ The majority of non-traumatic ICHs (primary $\mathrm{ICH}$ ) originate from the spontaneous rupture of small vessels damaged by chronic hypertension or amyloid angiopathy. ${ }^{3} \mathrm{ICH}$ is well-known for its high overall stroke mortality and disability. ${ }^{4}$ It carries a 1-year fatality of $>50 \%$, with only $20 \%$ of patients being independent at 6 months. ${ }^{4}$ The burden of lacunes, with other manifestations of CSVD, are a risk factor for disability, increased mortality, and unfavorable stroke outcomes. ${ }^{5}$ In addition to the ischemic and hemorrhagic strokes, cognitive impairment and gait disorders are frequently seen in older adults with underlying $\mathrm{CSVD}_{1}^{2,6,7}$ causing substantial disability. It is noteworthy that the epidemiology of CSVD might differ between Asian and Western populations, ${ }^{8,9}$ and the impact of CSVD may be even larger in non-Caucasian populations. ${ }^{10-12}$ In this review article, we aim to summarize recent advances in the prevention of primary $\mathrm{ICH}$ and $\mathrm{LI}$, as patients with CSVDs are at risk for both conditions. We discuss the latest advances in diagnosis and management with emphasis on antithrombotic use/avoidance and individualized vascular risk factor control.

\section{Cerebral small vessel diseases: major etiology of lacunar infarction and intracerebral hemorrhages}

CSVD includes a group of vascular pathologies with various etiologies that affect the small arteries, arterioles, venules, and capillaries of the brain, leading to both ischemic and hemorrhagic consequences. ${ }^{2}$ There are three common categories of vascular pathologies underlying CSVD.

\section{Hypertensive small vessel disease}

$\mathrm{LI}$, and hypertensive $\mathrm{ICH}$, mostly located in the brain's deep grey structure, such as the basal ganglia and thalamus, are mostly related to hypertensive (HTN)-SVD (Figure 1). The underlying small vessel pathologies are mainly characterized by arteriolosclerosis, most commonly in the form of fibrinoid necrosis and lipohyalinosis, and involvement of deep-seated vessels that stem directly from the large vessels as arterial perforators. ${ }^{2}$ Degenerative changes in the walls of the cerebral small vessels could cause ischemic Lls, microaneurysm formation, and $\mathrm{ICH}$. Other commonly coexisting systemic vascular risk factors, such as abnormal glucose metabolism, may also lead to impaired endothelial function and subsequently cSVD. ${ }^{13}$
However, the relationship between hyperlipidemia and CSVD is contradictory. A low low-density lipoprotein cholesterol (LDLC) level has been proposed as the potentially underlying mechanism for fragile endothelium, causing higher risks of vessel rupture and ICH. $.^{14} \mathrm{HTN}-\mathrm{SVD}$ has been associated with various additional radiological findings, including deep cerebral microbleeds (CMBs) (Figure 1B), white matter disease (leukoaraiosis) (Figure $1 \mathrm{C}$ ), and MRI-visible enlarged perivascular spaces (EPVS) (Figure 1F) in the basal ganglia. ${ }^{15,16}$ Recently, mixed-ICH, which refers to combined deep and lobar hemorrhage, is suggested to be associated with vascular risk factors similar to hypertensive $\mathrm{ICH}$, but demonstrates more severe parenchymal damage and higher $\mathrm{ICH}$ recurrence risk (Figure 1D). ${ }^{17,18}$

\section{Cerebral amyloid angiopathy}

Cerebral amyloid angiopathy (CAA) is characterized by the progressive accumulation of $\beta$-amyloid in the leptomeningeal and cortical vessels of the brain (Figure 2). ${ }^{19}$ Lobar ICH is the typical presentation of CAA-related vasculopathy. The association between hemorrhagic CSVD markers and CAA is well-recognized, including strictly lobar CMB (Figure 2B) and cortical superficial siderosis (CSS) (Figure 2D). ${ }^{20}$ The combination of at least one lobar ICH and either one or more strictly cortical/lobar CMB or CSS defines CAA in a patient older than 55 years old when other potential causes are ruled out. ${ }^{21,22}$ While most research has focused on its hemorrhagic manifestations, findings from radiologic and pathologic studies suggest that ischemic lesions from CAA also have substantial relevance. ${ }^{23}$ Recently, lacunes located in the centrum semiovale (CSO) or lobar regions, collectively called lobar lacunes (Figure 2E), were reported to be a potential CAA marker, ${ }^{24}$ further expanding the etiology of $\mathrm{LI}$ from chronic hypertension to a different vascular pathology. ${ }^{25}$

\section{Hereditary causes of cerebral small vessel diseases} A small, but significant proportion of small vessel diseases (SVDs) are of Mendelian inheritance (Figure 3). However, it is generally considered that the currently-identified monogenic causes represent less than 20\% of all monogenic forms of cSVD. ${ }^{26}$ Among these, Cerebral Autosomal Dominant Arteriopathy with Subcortical Infarcts and Leukoencephalopathy (CADA$\mathrm{SIL}$ ) is the most frequent and most well-known disorder, with hundreds to thousands of families diagnosed worldwide. ${ }^{27} \mathrm{CA}-$ DASIL is linked to stereotyped mutations of the neurogenic locus notch homolog protein 3 (NOTCH3) gene in chromosome $19 .^{28}$ The course of the disorder is now well known in its common form and rarer presentations are currently being recognized. MRI white matter hyperintensities (WMH) appear often before 30 years, and MRI results are usually abnormal at age 

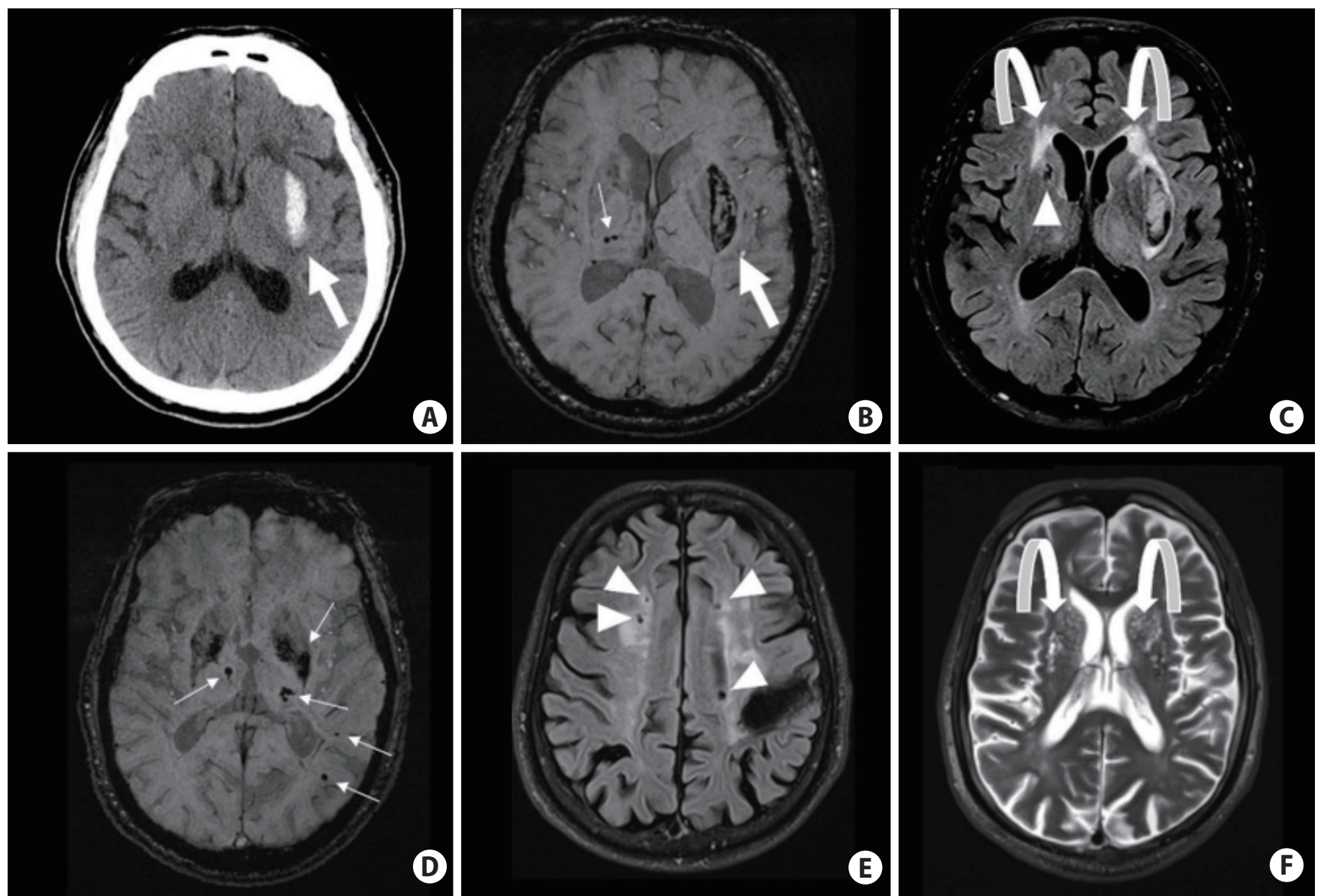

Figure 1. Computed tomography and magnetic resonance imaging (MRI) scans of hypertensive small vessel disease and associated bleeding risks. (A-C) Hypertensive intracerebral hemorrhage (ICH) in the left basal ganglia (big arrows); 1.6\% to 2\% annual recurrence risk. Several deep microbleeds (MBs) (small arrows), periventricular white matter hyperintensities (curved arrows) and a deep lacune (arrowhead) are also seen. (D-F) Mixed-location of ICH and MBs (small arrows), associated with $\sim 5.1 \%$ annual risk of $\mathrm{ICH}$ recurrence. There are several lacunes (arrowheads) and MRI-visible enlarged perivascular spaces near the basal ganglia (curved arrows) in the same patient.

40. In their forties or fifties, CADASIL patients present their first ischemic strokes related to small subcortical infarcts. However, approximately $30 \%$ of patients never suffer strokes. ${ }^{29}$ The number of strokes does not seem to markedly influence the disease course, which is generally more severe in men, for yet unknown reasons. ${ }^{30}$ The one modifiable risk factor is smoking, which multiplies ischemic stroke risk by a factor of three in these patients. ${ }^{31}$ While it was originally thought that most patients become demented and bedridden in their sixties, benign courses have been determined, patients (particularly women) in their seventies can be seen without any symptom. ${ }^{29}$

The second most frequent cause of hereditary cSVD is related to dominant HTRA1 mutations in chromosome 10, which are estimated to represent approximately $5 \%$ of hereditary cSVD. ${ }^{26,27}$ Given the recent characterization of its role in a form of dominantly inherited SVD, prospective data are lacking, thus far, to identify the disease characteristics. Most reported pa- tients presented with severe SVD in the elderly, sometimes in a context of uncontrolled hypertension. The pattern of WMH does not seem to be specific. ${ }^{26}$

Autosomal recessive forms related to mutations in the same gene led to the extremely rare Cerebral Autosomal Recessive Arteriopathy with Subcortical Infarcts and Leukoencephalopathy (CARASIL) disorder, diagnosed so far in a few tens of individuals, particularly in Asians ${ }^{32}$ and reports on Caucasian patients are exceptional. ${ }^{33}$ This disorder is characterized by spondylosis and alopecia in most cases, and most reports emphasize the severity of the disorder, often leading to disability, dementia, or death as soon as the fifth decade. ${ }^{34}$

The third most frequent form is related to autosomal dominant mutations in COLIVA1 or COLIVA2 genes, leading to different phenotypes that mostly predispose to $\mathrm{ICH}^{35}$ Many unknowns remain as illustrated by the presumed high frequency of mutation careers. The disease includes various extra-neurological fea- 

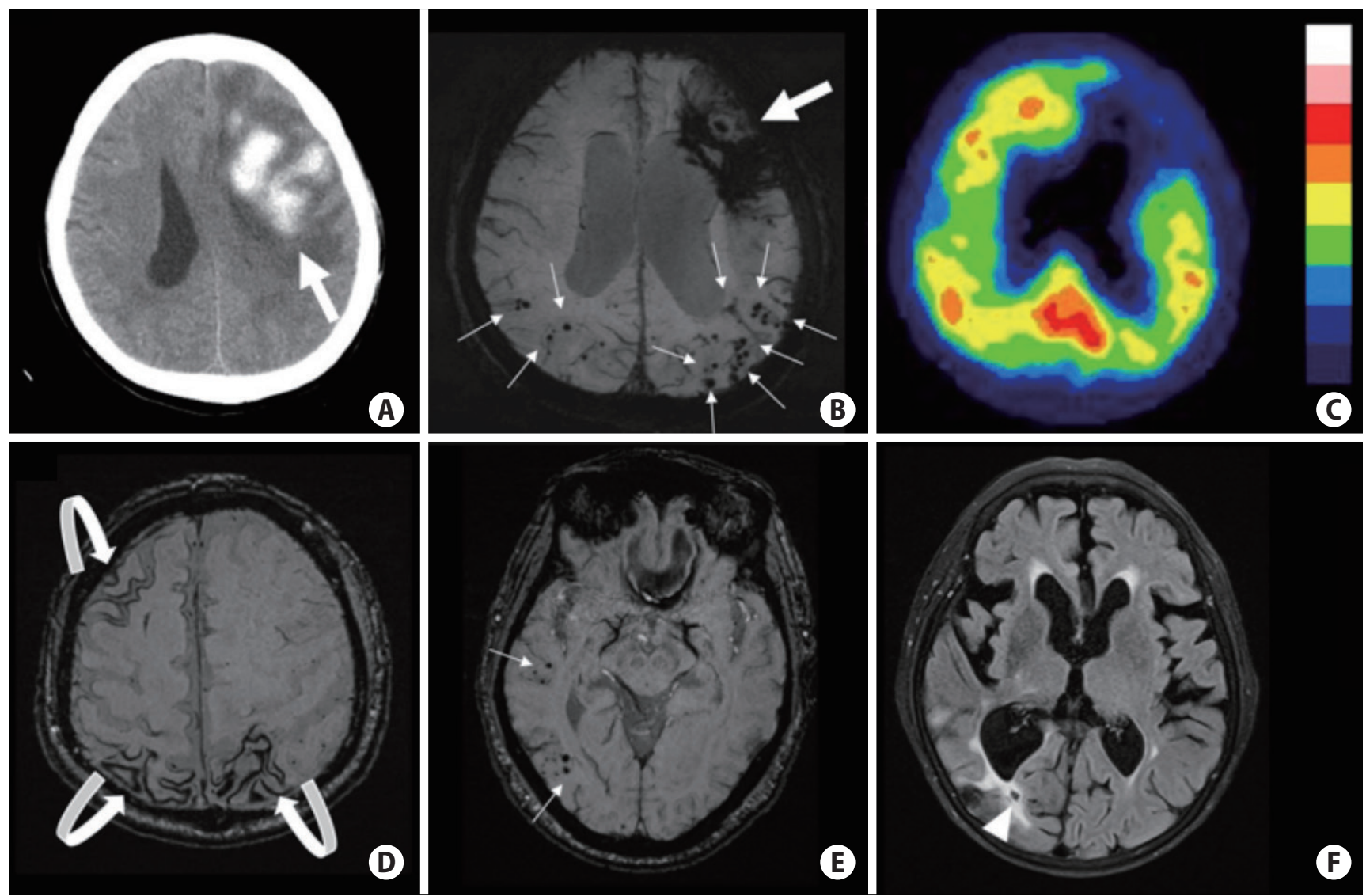

Figure 2. Computed tomography and magnetic resonance imaging scans of cerebral amyloid angiopathy (CAA) related pathologies and associated bleeding risk. (A, B) Probable CAA (per Boston criteria) with lobar intracerebral hemorrhage (ICH) (big arrows) and multiple cortical microbleeds (MBs) (small arrows); $\sim 10 \%$ annual ICH recurrence risk. (C) Increased tracer uptake detected on ${ }^{11} \mathrm{C}-$ Pittsburgh Compound B positron emission tomography scans in the same patient as A and B note the posterior predominance typical of CAA. (D) Multifocal cortical superficial siderosis (curved arrows) in a probable CAA patient who had ICH (not shown on this cut); 27\% annual risk of recurrent ICH. (E) CAA presenting with only cortical MBs (small arrows); 5\% yearly risk of first-time symptomatic ICH. (F) Lobar lacune (arrowhead) in a probable CAA patient who had ICH (not shown on this cut).

tures, including ocular, renal, and muscular manifestations.

A recently recognized phenotype corresponds to mutations of the promotor area of the COLIVA1 gene and lead to the pontine autosomal dominant microangiopathy and leukoencephalopathy (PADMAL) phenotype. In this rare disorder, small subcortical ischemic lesions and lacunes accumulate within the pons, with a clear predominance with respect to other brain regions. ${ }^{36}$ Other genes have been described, but the associated disorders are rarely encountered in clinical practice. In line with CADASIL and CARASIL, the acronym CARASAL has been proposed to describe a rare disorder related to autosomal mutations of the cathepsin A gene. ${ }^{37}$ Other forms, such as familial amyloid angiopathy, are also rarely seen. In comparison to sporadic forms, and in addition to familial history of CAA, the presence of calcifications of the cerebral cortex is quite specific. ${ }^{38}$ Hereditary forms of CAA cause even more frequent recurrent lobar ICHs starting in the 30 s to 40 s and cause structural brain injury in mutation careers even before the first $\mathrm{ICH}^{39,40}$
The diagnosis of CSVD is mainly based on lesions in the brain parenchyma mostly located in the subcortical structures. These lesions can be readily detected on MRI, including intraparenchymal hemorrhage, $\mathrm{CMB}, \mathrm{CSS}$, lacunes, $\mathrm{WMH}$, and MRI-visible EPVS. The differentiation between HTN-SVD and CAA is traditionally based on hemorrhage location. CAA-related ICH and CMB are located exclusively in the cortical/lobar regions, while hypertensive emorrhage is usually located in the deep parts of the brain. ${ }^{3,21,41}$ The presence of strictly lobar ICH/CMBs showed $100 \%$ accuracy for CAA in a radiologic/pathologic validation study. ${ }^{21} \mathrm{~A}$ cSS is another hemorrhagic feature of CAA and is typically absent in other types of CSVD. ${ }^{20,22,42}$ Similar to the distribution of hemorrhagic lesions, the topography of lacunes was shown in a recent study to be different between CAA and HTN-SVD, with lobar (or non-deep seated) lacunes being associated with CAA while deep lacunes were found to be more frequent with HTN-SVD. ${ }^{24}$ White matter disease is prominent in both HTN-SVD and CAA. ${ }^{43-48}$ Different WMH patterns between HTN-SVD and CAA have been proposed, but the signifi- 


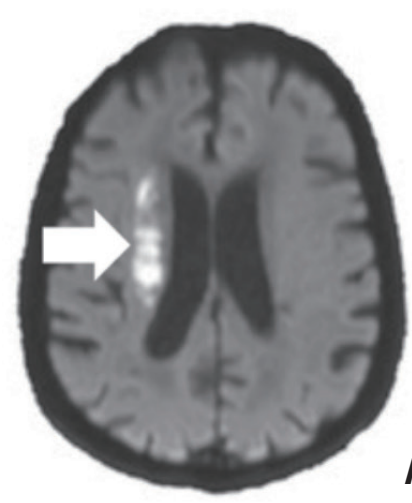

A

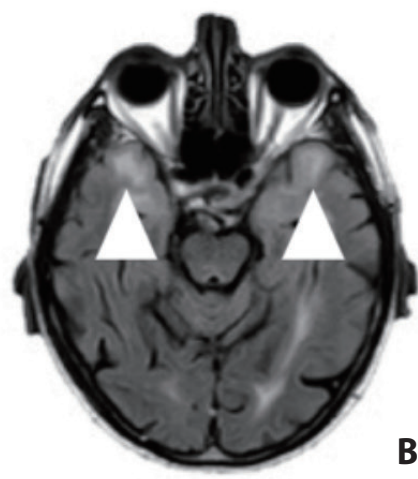

B

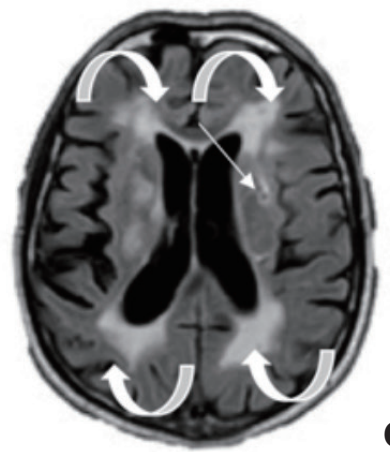

C

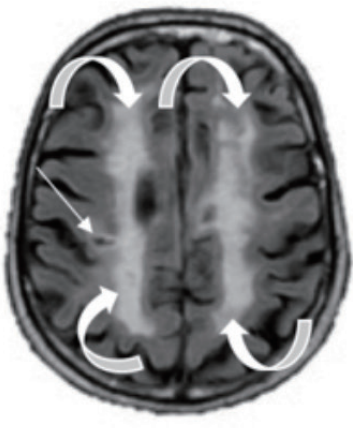

D

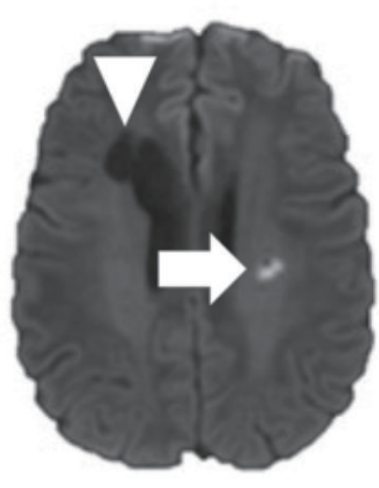

E

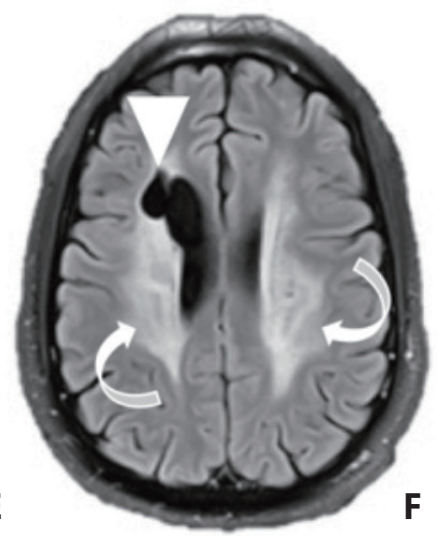

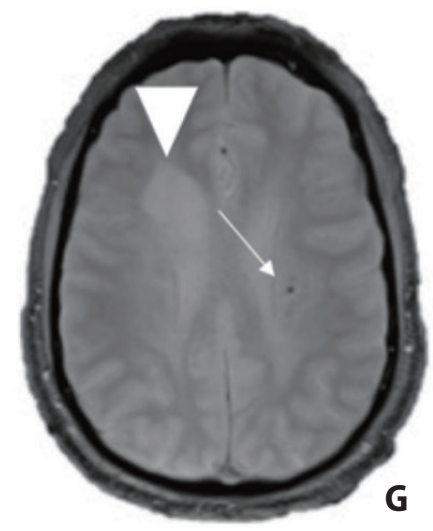

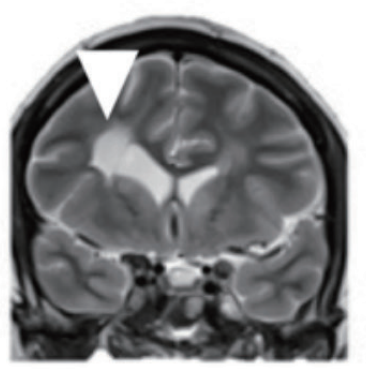

H

Figure 3. Neuroimaging characteristics of two patients with hereditary small vessel diseases (SVD). Top row: A 64-year-old male patient without medical history presented with acute left hemiplegia. (A) Imaging revealed an acute right deep-seated infarct (arrow) as well as (B, C) widespread white matter hyperintensities (curved arrows, arrowheads) and (C, D) multiple lacunes (small arrows). (B) Fluid attenuated inversion recovery (FLAIR) also shows bilateral anterior temporal white matter hyperintensities (arrowheads). The patient was diagnosed with Cerebral Autosomal Dominant Arteriopathy with Subcortical Infarcts and Leukoencephalopathy (CADASIL) based on genetic testing. Bottom row: a 32-year-old male patient presented with acute dysarthria related to a small subcortical infarct ( $E$, arrow). (F) Brain MRI showed otherwise diffuse white matter hyperintensities (curved arrows), (E-H) a right porencephalic lesion (arrowhead) and (G) microbleeds (small arrow). The patient was proven to have a COLIVA mutation.

cant overlap in WMH patterns suggests that leukoaraiosis is probably multifactorial in many cases.$^{45} \mathrm{MRI}$-visible EPVS, which might imply cerebrospinal fluid (CSF) drainage impairment, has also been suggestive of an important marker in cSVD. Previous studies have showed that severe EPVS in the basal ganglia was associated with HTN-SVD, while high-degree EPVS in CSO was linked to CAA. ${ }^{16,49}$

In addition to radiological markers, a decreased amyloid $\beta$ $(A \beta)$ level in the $C S F$, particularly $A \beta 40$ and $A \beta 42$, may serve as a molecular biomarker to identify CAA patients. ${ }^{50}$ Another important advancement is the application of amyloid positron emission tomography (PET) using ${ }^{11} \mathrm{C}$-Pittsburgh Compound $\mathrm{B}$ or ${ }^{18}$ F-Florbetapir. ${ }^{6,51}$ Amyloid PET provides a noninvasive method to directly quantify $\beta$-amyloid presence and burden in vivo (Figure $2 \mathrm{C}$ ). Given that it labels both cerebrovascular and parenchymal amyloid, amyloid-PET appears to have better diagnostic accuracy under appropriate clinical settings, such as in differentiating patients with probable CAA from cognitively normal healthy controls or patients with deep $\mathrm{ICH}^{.51}$

The identification of hereditary causes is of high interest in patients with a history of stroke. First, when natural history is well described, there is an opportunity to inform patients, given that the fate of the disease might be different from that of sporadic forms. Currently, this applies mostly to CADASIL, for which benign forms have been regularly observed. In addition, modifiable factors may be known to influence the disease course and controlling such risks is important. For instance, smoking is strongly associated with stroke recurrence in CADASIL, while it is clearly known that even minor head trauma can trigger devastating ICH in COLIVA1 patients. The asymptomatic forms present unique challenges. In the absence of any curative treatment, the pros and cons of prescribing a genetic test should be discussed with the patients. In a major center dealing with a high volume of hereditary cSVD such as Lariboisière Hospital, asymptomatic individuals must undergo a multi-professional consultation before genetic testing can be performed ${ }^{52}$ 
By contrast to what was previously thought, it seems that no aspect of imaging is specific to any hereditary etiology. For long, it has been considered that anterior temporal pole hyperintense lesions, as well as that of the superior frontal gyrus and subinsular areas, were specific to CADASIL, ${ }^{53}$ but these aspects might be also observed in sporadic forms. It is possible that they are more frequent in hereditary causes including NOTCH3 negative forms. Other aspects might be more often observed in some cases. For instance, an état criblée of the basal ganglia may be more prevalent in HTRA 1 autosomal dominant related forms. ${ }^{26}$ In addition, multiple small infarcts and lacunes of the pons, contrasting with a relative preservation of other brain areas, is in favor of mutations of the promotor of COLIVA1 (the PADMAL phenotype).$^{36}$ However, given the diversity of clinicoradiological presentations of CSVD, even within families affected by the same disorder, none of these aspects are very helpful. By contrast, a discrepancy between a low risk factor profile and a severe clinicoradiological presentation is helpful, particularly when familial history is lacking.

Diagnosing the underlying vascular pathology is crucial because there is a significantly different risk of recurrent ICH between CAA and HTN-SVD (Figures 1 and 2). Patients who suffer from CAA-related ICH carry around 10\% of an annual ICH recurrence risk. Such risk is even higher if $\mathrm{CSS}$ is present, and increases up to $27 \%$ annually with widespread cSS. ${ }^{20,54}$ Patients with multiple strictly lobar CMB (without associative ICH) had risk factors and neuroimaging profiles similar to $C A A$, and possess a $5 \%$ annual risk of developing first-time symptomatic ICH during follow-up. ${ }^{54}$ In contrast, the ICH risk is reported as $2 \%$ per year in survivors of hypertensive $\mathrm{ICH}^{17,55}$ In patients with mixed-ICH (Figure 1E), which may represent the consequence of more severe underlying HTN-SVD, the ICH recurrence rate increases to $5.1 \%$ per year according to a recent study. ${ }^{17}$ Thus, identifying the underlying microangiopathy is crucial, especially in situations where patients have an indication for antithrombotic use, to balance hemorrhagic versus ischemic risks. The diagnosis may further influence clinical decisions regarding management of vascular risk factors, especially considering statin use for hyperlipidemia. ${ }^{14}$

The ICH risk seems very low in Caucasian patients with CADASIL. Among 278 CADASIL patients from the Paris-Munich cohort followed for 3 years, 55 strokes occurred and all were ischemic; no ICH was observed. ${ }^{31}$ Even more recently, 69 ischemic strokes were observed but no ICH among 369 patients followed for a median of 3.9 years, leading to an estimated average incidence of ischemic stroke of 4.4 per 100 people per year. ${ }^{56}$ The ischemic stroke risk was higher in patients with microbleeds than in those without, but all predictors were other- wise equal..$^{56}$ While rare ICH in hereditary cSVD has been clearly documented in Western countries, ICH risk might be far larger in Asian populations. In a cohort of 94 CADASIL patients from Korea, 16 had an $\mathrm{ICH}^{57} \mathrm{ICH}$ risk seems to be strongly linked to hypertension, ${ }^{58}$ which might be more prevalent in Asian populations. To our knowledge, there is no data on estimations of ICH risk or ischemic stroke recurrence in patients with COLIVA1 mutations or other rare hereditary CSVD.

In summary, patients who have any combination of these vascular pathologies or associated parenchymal lesions are at risk for both ischemic and hemorrhagic stroke. Recent improvements in understanding the above-mentioned radiological markers for sporadic CSVD can help the clinician stratify ICH risk in individual patients. There are general good practice measures for stroke prevention in patients with CSVD, while individualized measures tailored for each patient are vital. These approaches will be further discussed in the subsequent sessions.

\section{Use or avoidance of antithrombotics for prevention of ICH and Lls}

Recurrent strokes in patients with previous $\mathrm{LI}$ or ICH are common. As discussed above, some ICH survivors might have ischemic strokes, and those with LI have an increased risk for recurrent ICH. ${ }^{59-61}$ Although antithrombotics play an important role in ischemic stroke prevention, determining the appropriate secondary prevention after $\mathrm{LI}$ is a therapeutic dilemma, especially knowing that ICH frequently causes devastating outcomes and is the most feared complication of antithrombotics. Clinical decision making should always be based on the assessment of ischemic stroke reduction weighed against hemorrhagic risks associated with antithrombotic use in patients with underlying CSVD. Oral anticoagulants are not considered for cSVD-related stroke prevention, including recurrent LIs, as they disproportionately increase $\mathrm{ICH}$ risk. Patients with hemorrhagic CSVD markers are at a particularly high risk for developing ICH under anticoagulation, and the use of such medications should be prevented, even when indicated for a concurrent pathology when alternatives exist, such as left atrial appendage closure in non-valvular atrial fibrillation. . 5,62,63 $^{2}$

Although studies focusing on $\mathrm{LI}$ are rare, the benefits of aspirin or other antiplatelets are considered to be similar between lacunar and non-Lls based on subgroup analyses of randomized controlled trials (RCTs) in all types of ischemic strokes. ${ }^{64}$ Aspirin and clopidogrel compared with clopidogrel alone after recent ischaemic stroke or transient ischaemic attack in high-risk patients (MATCH) trial revealed higher incidences of major bleeding in patients with long-term dual anti- 
platelet therapy (75 mg clopidogrel and $75 \mathrm{mg}$ aspirin combination vs. $75 \mathrm{mg}$ clopidogrel). ${ }^{65}$ The Secondary Prevention of Small Subcortical Strokes (SPS3) trial is the only study focusing on the efficacy of antiplatelet agents in stroke prevention for patients with $\mathrm{LI}^{60}{ }^{60}$ This study was a RCT that included 3,020 patients who were randomized to receive aspirin monotherapy (325 mg aspirin) or dual antiplatelet therapy (325 mg aspirin and $75 \mathrm{mg}$ clopidogrel). Dual antiplatelet therapy was found to increase the incidence of major hemorrhage and mortality rate, and thus, the trial was stopped early. Moreover, the risk for recurrent stroke was not significantly reduced by dual antiplatelet therapy. Overall, these studies showed that dual antiplatelet therapy might increase major bleeding risk without providing additional stroke reduction benefits for Lls. Currently, antiplatelet monotherapy is recommended to prevent recurrent strokes after LI. ${ }^{64}$ Cilostazol and triflusal, while offering similar efficacies in stroke prevention, may have lower risks of hemorrhage than aspirin in recent studies. ${ }^{66,67}$ The subgroup analysis of cilostazol for prevention of secondary stroke (CSPS2) study in patients with LI (100 mg cilostazol twice a day vs. 81 mg aspirin once a day) showed that the incidence of ICH was significantly lower in patients using cilostazol than aspirin alone. ${ }^{68}$ In a recent trial for the prevention of cardiovascular events in Asian patients with ischemic stroke at high risk of cerebral haemorrhage (PICASSO) ${ }_{1}^{69}$ authors enrolled 1,534 patients with ischemic stroke and a history of or imaging findings of $\mathrm{ICH}$ or two or more microbleeds, and randomized them to receive either aspirin or cilostazol treatments. During a median followup of 1.9 years, the incidence of $\mathrm{ICH}$ was 0.61 per 100 people per year in patients who received cilostazol and 1.20 per 100 people per year in those who received aspirin (hazard ratio [HR], 0.51; 97.5\% confidence interval [Cl], 0.20 to 1.27; superiority $P=0.18$ ). Although the results are not statistically significant, they are consistent with previous reports from Asia, and suggest that cilostazol may be an antiplatelet suitable for patients with ischemic stroke at increased ICH risk. However, cilostazol research has been scarce in non-Asian patients, and further large-scale studies are warranted to validate the results in non-Asian populations. ${ }^{70}$

CSVD patients with a concomitant cardioembolic source or other problems for whom anticoagulation would normally be used present a therapeutic dilemma. Life-long anticoagulation is contraindicated after cSVD-related ICH, and should also be avoided to the extent possible in patients with cSVD-related lacunar strokes, given that anticoagulants may lead to increased major bleeding risks in such patients. ${ }^{63,71-73}$ All of the phase three trials of the four non-vitamin $\mathrm{K}$ oral anticoagulants have excluded patients with a history of $\mathrm{ICH}$ or those with a high risk for hemorrhage. ${ }^{74}$ For patients without $\mathrm{ICH}$ that have subtle hemorrhagic lesions, such as CMB and cSS, the use of long-term anticoagulation is also problematic. ${ }^{63,75,76}$

Nonpharmacological approaches, such as left atrial appendage closure, might provide potential alternatives to life-long anticoagulation for non-valvular atrial fibrillation and are further discussed in an accompanying review article in this issue of the Journal of Stroke as well as in a separate recent review. ${ }^{63,77}$ In acute myocardial infarction and deep venous thrombosis, anticoagulation time should be minimized to the lowest extent possible if the patient has CSVD. A careful riskbenefit assessment is always needed in individual patients after stratifying the $\mathrm{ICH}$ risks (summarized in the previous parts and figures). Patients at a low ICH risk might tolerate anticoagulation for shorter timeframes. ${ }^{78}$

\section{Individualized optimal control of vascular risk factors}

\section{Hypertension}

Hypertension is the best known and prevalent risk factor for stroke, especially for cSVD-related $\mathrm{LI}$ and $\mathrm{ICH}^{79}$ Prolonged exposure of intracranial arterioles to hypertension can be associated with endothelial dysfunction, ultimately causing CSVD. ${ }^{80}$ Although correction of hypertension is an established way for stroke prevention, the optimum blood pressure (BP) in patients with CSVD has been unknown; decreased cerebral perfusion might be expected under low BP states if autoregulation was chronically damaged.

The perindopril protection against recurrent stroke study (PROGRESS) trial reported a reduction in BP by $9 / 4 \mathrm{~mm} \mathrm{Hg}$ corresponding to a reduction in recurrent stroke by $28 \%$ ( $95 \%$ $\mathrm{Cl}, 17 \%$ to $38 \%$ ) compared with placebo during a mean follow-up of 3.9 years in 6,105 patients with previously symptomatic cerebrovascular disease; however, there was no data regarding patients with a qualifying event of LI. ${ }^{81}$ In the Prevention Regimen for Effectively Avoiding Second Strokes (PRoFESS) trial, a reduction in BP by $3.8 / 2.0 \mathrm{~mm} \mathrm{Hg}$ corresponded to a nonsignificant reduction in recurrent stroke by $5 \%(95 \%$ $\mathrm{Cl},-4 \%$ to $14 \%$ ) compared with the placebo during a mean follow-up of 2.5 years in 20,332 patients with recent ischemic stroke, in which $52 \%$ of the qualifying strokes were LI. ${ }^{82}$ The SPS3 trial is the only RCT to test the target BP level for secondary prevention in patients with symptomatic LI. This study included 3,020 patients and compared two systolic BP targets of 130 to $149 \mathrm{~mm} \mathrm{Hg}$ versus $<130 \mathrm{~mm} \mathrm{Hg}$ with a mean followup of 3.7 years. There was a $19 \%\left(95 \% \mathrm{Cl}_{1}-3 \%\right.$ to $\left.36 \%\right)$ nonsignificant reduction in recurrent stroke, but the lower BP tar- 
get group ( $<130 \mathrm{~mm} \mathrm{Hg}$ ) had markedly reduced ICH risks (HR, $0.37 ; 95 \% \mathrm{Cl}, 0.14$ to 0.89$).{ }^{83}$ Thus far, there is no RCT regarding $\mathrm{BP}$ control for secondary prevention in ICH survivors; however, in a single-center observational study, inadequate BP control was associated with a higher lobar and non-lobar ICH recurrence risk. ${ }^{84}$ Therefore, current evidence suggests that more stringent BP control (within or close to normal limits) should be favored over less strict control for stroke prevention in patients harboring $\mathrm{CSVDs}{ }^{85-87}$

While there is no proof that BP lowering is efficient for stroke prevention in hereditary CSVD, there are indirect arguments suggesting a reduction in $\mathrm{ICH}$ risk with strict BP lowering in CADASIL patients. However, it is important not to lower BP excessively, and particular attention is needed for specific situations, such as general anesthesia, because some reports have clearly highlighted a risk of multiple concurrent small subcortical infarcts. ${ }^{88}$ In COLIVA1 patients, BPs are monitored and normotension is actively targeted with antihypertensive medication use when needed.

\section{Diabetes}

Both diabetes mellitus and metabolic syndrome are related to increased incidence of ischemic stroke and ICH, and to the risk of recurrent strokes in patients with LI. ${ }^{89-92}$ In the SPS3 trial, which specifically included patients with symptomatic LI, diabetic patients were almost twice as likely to have a recurrent, especially ischemic, stroke ( $\mathrm{HR}, 1.8 ; 95 \% \mathrm{Cl}, 1.4$ to 2.4$)$. Diabetic patients also had more frequent intracranial stenosis, infarcts involving posterior circulation, and more extensive $\mathrm{WMH} .{ }^{93}$ Data on blood sugar control and ICH risk remains limited. In a community-based prospective cohort study in China with 96,110 patients, either low $(<4.0 \mathrm{mmol} / \mathrm{L}$ or $72 \mathrm{mg} / \mathrm{dL}$ ) or high ( $\geq 6.1$ $\mathrm{mmol} / \mathrm{L}$ or $110 \mathrm{mg} / \mathrm{dL}$ ) fasting blood glucose levels led to elevated incident ICH risk in a median follow-up of 9 years. ${ }^{13}$ Keeping patients euglycemic to the extent possible and preventing hypoglycemia might be potentially beneficial in CSVD prevention, but these approaches need to be further validated.

\section{Hyperlipidemia}

Hyperlipidemia, particularly elevated LDL-C levels, is an important risk factors for ischemic stroke and is also a general target for secondary preventive measures. However, there are concerns regarding possible associations between low lipoprotein levels and statin use on $\mathrm{ICH}$ risk. ${ }^{14}$ The Stroke Prevetion by Aggressive Reduction in Cholestrol Levels (SPARCL) trial demonstrated the efficacy of lipid-lowering therapy in patients with a recent ( $<6$ months) transient ischemic attack or stroke and a baseline cholesterol level between 100 and $190 \mathrm{mg} / \mathrm{dL}$. In this trial, atorvastatin reduced the incidence of recurrent stroke, from $13.1 \%$ to $11.2 \%$ over a 5 -year follow-up period. ${ }^{94}$ However, patients with $\mathrm{ICH}$ who received statins were found to have a significantly increased $\mathrm{ICH}$ recurrence risk. ${ }^{95,96} \mathrm{~A}$ post hoc analysis of the SPARCL trial shows a significantly increased $\mathrm{ICH}$ risk in patients with baseline CSVD-related $\mathrm{LI}$ who received statins (2.8\% vs. $0.6 \% ; \mathrm{HR}, 4.99 ; 95 \% \mathrm{Cl}, 1.71$ to 14.61$)$, but also shows a counterbalancing trend toward benefit for ischemic stroke reduction (11.2\% vs. $14.6 \%$; HR, $0.76 ; 0.57$ to 1.02). ${ }^{96}$ Benefits and potential risks of statin use should be explicitly discussed with all patients who might have a higher hemorrhagic risk and an algorithm for statin treatment in this context was published in a recent review article. ${ }^{14}$

\section{Homocysteine elevation and its treatment}

Homocysteine promotes the endothelial inflammatory response and oxidative stress-induced endothelial damage, and hyperhomocysteinemia is a risk factor for CSVD. ${ }^{97}$ The availability and the low side effect profile of a multivitamin supplementation for hyperhomocysteinemia treatment (oral folic acid, B6and B12-vitamins), might provide a favorable addition to the standard preventive measures. ${ }^{98}$ In the VITAmins TO Prevent Stroke (VITATOPS) trial, homocysteine-lowering therapy and Bvitamins were associated with a significant reduction in $\mathrm{WMH}$ volume change in those with severe baseline CSVD.99

\section{Renal failure, hepatic failure, and other systemic} vascular risk factors

CSVD has been reported to correlate strongly with chronic kidney disease. Decreased creatinine clearance appears to be a contributing factor to silent LIs, and even possibly to other types of cSVD-related lesions, such as WMH and CMB. ${ }^{100}$ Chronic hepatic dysfunction and other systemic diseases have also been linked to CSVD and ICH. Therefore, it is important to optimize the function of other organ systems to the extent possible to minimize cSVD risks.

\section{Specific considerations}

\section{Amyloid lowering for CAA}

Preventive treatment for CAA is currently limited, but there are growing interest and international efforts to identify potential candidates that inhibit the formation or deposition of $\beta$-amyloid. Only one phase two trial with tramiprosate, an oral agent that decreases vascular $A \beta$ burden in the transgenic mouse models, was published and confirmed its safety. ${ }^{101}$ Thus far, there is no specific therapy shown to lower ICH recurrence in CAA in humans. 


\section{Conclusions}

Lls and intraparenchymal hemorrhages share a common etiology-the cSVDs in most cases. These conditions are mostly sporatic, but a better understanding of hereditary forms is developing, resulting in an accurate diagnosis of the latter. Several good practice measures, such as optimal BP and diabetes management, can help reduce the risk of both stroke types in patients with SVDs. Antiplatelet combinations and anticoagulants have no specific role in ischemic lacunar stroke prevention and increase the risk of usually fatal/disabling brain bleeds in these patients. A good understanding of parenchymal hemorrhage risk is crucial for appropriate management, especially for use/avoidance of antithrombotics; such risk stratification can be monitored using available MRI markers.

\section{Disclosure}

The authors have no financial conflicts of interest.

\section{Acknowledgments}

This work was made possible by grants from the National Institute of Health (M. Edip Gurol, NS083711).

\section{References}

1. Adams HP Jr, Bendixen BH, Kappelle $\sqcup$, Biller J, Love BB, Gordon DL, et al. Classification of subtype of acute ischemic stroke. Definitions for use in a multicenter clinical trial. TOAST. Trial of Org 10172 in Acute Stroke Treatment. Stroke 1993;24:35-41.

2. Pantoni L. Cerebral small vessel disease: from pathogenesis and clinical characteristics to therapeutic challenges. Lancet Neurol 2010;9:689-701.

3. Qureshi Al, Tuhrim S, Broderick JP, Batjer HH, Hondo H, Hanley DF. Spontaneous intracerebral hemorrhage. $N$ Engl J Med 2001;344:1450-1460.

4. Gurol ME, Greenberg SM. Management of intracerebral hemorrhage. Curr Atheroscler Rep 2008;10:324-331.

5. Song TJ, Kim J, Song D, Yoo J, Lee HS, Kim YJ, et al. Total cerebral small-vessel disease score is associated with mortality during follow-up after acute ischemic stroke. $J$ Clin Neurol 2017;13:187-195.

6. Gurol ME. Molecular neuroimaging in vascular cognitive impairment. Stroke 2016;47:1146-1152.

7. de Laat $K F$, van Norden $A G$, Gons RA, van Oudheusden $\sqcup$, van Uden IW, Bloem BR, et al. Gait in elderly with cerebral small vessel disease. Stroke 2010;41:1652-1658.

8. Fanning JP, Wong AA, Fraser JF. The epidemiology of silent brain infarction: a systematic review of population-based cohorts. BMCMed 2014;12:119.

9. Wolma J, Nederkoorn PJ, Goossens A, Vergouwen MD, van Schaik IN, Vermeulen M. Ethnicity a risk factor? The relation between ethnicity and large- and small-vessel disease in White people, Black people, and Asians within a hospitalbased population. Eur J Neurol 2009;16:522-527.

10. Mok V, Kim JS. Prevention and management of cerebral small vessel disease. J Stroke 2015;17:111-122.

11. Venketasubramanian N, Yoon BW, Pandian J, Navarro JC. Stroke epidemiology in South, East, and South-East Asia: a review. J Stroke 2017;19:286-294.

12. An SJ, Kim TJ, Yoon BW. Epidemiology, risk factors, and clinical features of intracerebral hemorrhage: an update. J Stroke 2017;19:3-10.

13. Jin C, Li G, Rexrode KM, Gurol ME, Yuan X, Hui Y, et al. Prospective study of fasting blood glucose and intracerebral hemorrhagic risk. Stroke 2018;49:27-33.

14. Lauer $A$, Greenberg $S M$, Gurol ME. Statins in intracerebral hemorrhage. Curr Atheroscler Rep 2015;17:46.

15. Breteler MM, van Swieten JC, Bots ML, Grobbee DE, Claus JJ, van den Hout JH, et al. Cerebral white matter lesions, vascular risk factors, and cognitive function in a population-based study: the Rotterdam Study. Neurology 1994;44:1246-1252.

16. Charidimou A, Boulouis G, Pasi M, Auriel E, van Etten ES,

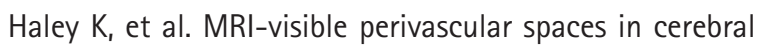
amyloid angiopathy and hypertensive arteriopathy. Neurology 2017;88:1157-1164.

17. Pasi M, Charidimou A, Boulouis $G$, Auriel $E$, Ayres A, Schwab $\mathrm{KM}$, et al. Mixed-location cerebral hemorrhage/microbleeds: underlying microangiopathy and recurrence risk. Neurology 2018;90:e119-e126.

18. Smith EE, Romero JR. Mixed emotions: what to do with patients who have lobar and deep hemorrhages on MRI? Neurology 2018;90:55-56.

19. Gurol ME, Greenberg SM. Cerebral amyloid angiopathies. In: Caplan LR, Biller J. Uncommon Causes of Stroke. 3rd ed. Cambridge, UK: Cambridge University Press, 2018:534-544.

20. Charidimou A, Boulouis G, Roongpiboonsopit D, Auriel E, Pasi $M$, Haley $K$, et al. Cortical superficial siderosis multifocality in cerebral amyloid angiopathy: a prospective study. Neurology 2017;89:2128-2135.

21. Knudsen KA, Rosand J, Karluk D, Greenberg SM. Clinical diagnosis of cerebral amyloid angiopathy: validation of the Boston criteria. Neurology 2001;56:537-539.

22. Linn J, Halpin A, Demaerel P, Ruhland J, Giese AD, Dichgans 
$M$, et al. Prevalence of superficial siderosis in patients with cerebral amyloid angiopathy. Neurology 2010;74:1346-1350.

23. Reijmer YD, van Veluw SJ, Greenberg SM. Ischemic brain injury in cerebral amyloid angiopathy. I Cereb Blood Flow Metab 2016;36:40-54.

24. Pasi M, Boulouis G, Fotiadis P, Auriel E, Charidimou A, Haley $\mathrm{K}$, et al. Distribution of lacunes in cerebral amyloid angiopathy and hypertensive small vessel disease. Neurology 2017; 88:2162-2168.

25. Smith $\mathrm{EE}$, Lee JM. Lacunes: black holes in our understanding of cerebral amyloid angiopathy. Neurology 2017;88:21582159.

26. Verdura $E_{1}$ Hervé $D$, Scharrer $E$, Amador Mdel M, GuyantMaréchal L, Philippi A, et al. Heterozygous HTRA1 mutations are associated with autosomal dominant cerebral small vessel disease. Brain 2015;138(Pt 8):2347-2358.

27. Di Donato I, Bianchi S, De Stefano N, Dichgans M, Dotti MT, Duering $\mathrm{M}_{1}$ et al. Cerebral Autosomal Dominant Arteriopathy with Subcortical Infarcts and Leukoencephalopathy (CADA$\mathrm{SIL}$ ) as a model of small vessel disease: update on clinical, diagnostic, and management aspects. BMCMed 2017;15:41.

28. Joutel A, Corpechot C, Ducros A, Vahedi K, Chabriat H, Mouton $\mathrm{P}$, et al. Notch3 mutations in CADASIL, a hereditary adult-onset condition causing stroke and dementia. Nature 1996;383:707-710.

29. Chabriat $H$, Joutel $A$, Dichgans $M$, Tournier-Lasserve $E$, Bousser MG. Cadasil. Lancet Neurol 2009;8:643-653.

30. Gunda $B$, Hervé $D$, Godin $O$, Bruno $M$, Reyes $S$, Alili N, et al. Effects of gender on the phenotype of CADASIL. Stroke 2012; 43:137-141.

31. Chabriat $H_{\text {, Hervé }} \mathrm{D}$, Duering $\mathrm{M}$, Godin $\mathrm{O}$, Jouvent $\mathrm{E}_{1}$ Opherk $\mathrm{C}$, et al. Predictors of clinical worsening in cerebral autosomal dominant arteriopathy with subcortical infarcts and leukoencephalopathy: prospective cohort study. Stroke 2016;47:4-11.

32. Nozaki $H$, Sekine $Y$, Fukutake T, Nishimoto $Y$, Shimoe $Y$, Shirata $A$, et al. Characteristic features and progression of abnormalities on MRI for CARASIL. Neurology 2015;85:459463.

33. Mendioroz M, Fernández-Cadenas I, Del Río-Espinola A, Rovira $A$, Solé $E$, Fernández-Figueras $M T$, et al. A missense HTRA1 mutation expands CARASIL syndrome to the Caucasian population. Neurology 2010;75:2033-2035.

34. Nishimoto $Y$, Shibata $M$, Nihonmatsu $M$, Nozaki $H_{\text {, Shiga } A}$ Shirata $A$, et al. A novel mutation in the HTRA1 gene causes CARASIL without alopecia. Neurology 2011;76:1353-1355.

35. Plaisier E, Gribouval O, Alamowitch $\mathrm{S}_{1}$ Mougenot B, Prost $\mathrm{C}_{1}$ Verpont MC, et al. COL4A1 mutations and hereditary angiopathy, nephropathy, aneurysms, and muscle cramps. N Engl
J Med 2007;357:2687-2695.

36. Verdura $E$, Hervé $D$, Bergametti $F$, Jacquet $C$, Morvan T, Prie-

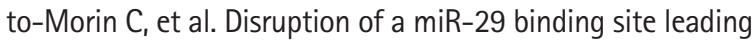
to COL4A1 upregulation causes pontine autosomal dominant microangiopathy with leukoencephalopathy. Ann Neurol 2016;80:741-753.

37. Bugiani M, Kevelam SH, Bakels HS, Waisfisz Q, Ceuterick-de Groote C, Niessen HW, et al. Cathepsin A-related arteriopathy with strokes and leukoencephalopathy (CARASAL). Neurology 2016;87:1777-1786.

38. Sellal F, Wallon D, Martinez-Almoyna L, Marelli C, Dhar A, Oesterlé $H$, et al. APP mutations in cerebral amyloid angiopathy with or without cortical calcifications: report of three families and a literature review. J Alzheimers Dis 2017;56:3746.

39. Fotiadis P, van Rooden $\mathrm{S}$, van der Grond J, Schultz A, Martinez-Ramirez $S$, Auriel $E$, et al. Cortical atrophy in patients with cerebral amyloid angiopathy: a case-control study. Lancet Neurol 2016;15:811-819.

40. van Etten ES, Gurol ME, van der Grond J, Haan J, Viswanathan A, Schwab KM, et al. Recurrent hemorrhage risk and mortality in hereditary and sporadic cerebral amyloid angiopathy. Neurology 2016;87:1482-1487.

41. Martinez-Ramirez S, Romero JR, Shoamanesh A, McKee AC, Van Etten $E$, Pontes-Neto O, et al. Diagnostic value of lobar microbleeds in individuals without intracerebral hemorrhage. Alzheimers Dement 2015;11:1480-1488.

42. Wollenweber FA, Baykara E, Zedde M, Gesierich B, Achmüller $M$, Jouvent $E$, et al. Cortical superficial siderosis in different types of cerebral small vessel disease. Stroke 2017;48:14041407.

43. Gurol ME, rrizarry MC, Smith EE, Raju S, Diaz-Arrastia R, Bottiglieri $\mathrm{T}$, et al. Plasma beta-amyloid and white matter lesions in $A D, M C l$, and cerebral amyloid angiopathy. Neurology 2006;66:23-29.

44. Gurol ME, Viswanathan A, Gidicsin C, Hedden T, MartinezRamirez S, Dumas A, et al. Cerebral amyloid angiopathy burden associated with leukoaraiosis: a positron emission tomography/magnetic resonance imaging study. Ann Neurol 2013;73:529-536.

45. Charidimou A, Boulouis G, Haley K, Auriel E, van Etten ES, Fotiadis $\mathrm{P}$, et al. White matter hyperintensity patterns in cerebral amyloid angiopathy and hypertensive arteriopathy. Neurology 2016;86:505-511.

46. Smith EE, Gurol ME, Eng JA, Engel CR, Nguyen TN, Rosand J, et al. White matter lesions, cognition, and recurrent hemorrhage in lobar intracerebral hemorrhage. Neurology 2004; 63:1606-1612. 
47. Chen YW, Gurol ME, Rosand J, Viswanathan A, Rakich SM, Groover TR, et al. Progression of white matter lesions and hemorrhages in cerebral amyloid angiopathy. Neurology 2006;67:83-87.

48. Smith EE, Nandigam KR, Chen $Y W_{1}$ Jeng J, Salat $D$, Halpin $A$, et al. MRI markers of small vessel disease in lobar and deep hemispheric intracerebral hemorrhage. Stroke 2010;41:19331938.

49. Martinez-Ramirez $S$, Pontes-Neto OM, Dumas AP, Auriel $E_{1}$ Halpin A, Quimby $M$, et al. Topography of dilated perivascular spaces in subjects from a memory clinic cohort. Neurology 2013;80:1551-1556.

50. Verbeek MM, Kremer BP, Rikkert MO, Van Domburg PH, Skehan $\mathrm{ME}$, Greenberg SM. Cerebrospinal fluid amyloid beta(40) is decreased in cerebral amyloid angiopathy. Ann Neurol 2009;66:245-249.

51. Gurol ME, Becker JA, Fotiadis P, Riley G, Schwab K, Johnson $K A$, et al. Florbetapir-PET to diagnose cerebral amyloid angiopathy: a prospective study. Neurology 2016;87:2043-2049.

52. Reyes $S$, Kurtz A, Hervé D, Tournier-Lasserve $E_{1}$ Chabriat $H$. Presymptomatic genetic testing in CADASIL. J Neurol 2012;259:2131-2136.

53. Auer DP, Pütz B, Gössl C, Elbel G, Gasser T, Dichgans M. Differential lesion patterns in CADASIL and sporadic subcortical arteriosclerotic encephalopathy: MR imaging study with statistical parametric group comparison. Radiology 2001;218:443451.

54. van Etten ES, Auriel E, Haley KE, Ayres AM, Vashkevich $A$, Schwab KM, et al. Incidence of symptomatic hemorrhage in patients with lobar microbleeds. Stroke 2014;45:2280-2285.

55. Thon JM, Gurol ME. Intracranial hemorrhage risk in the era of antithrombotic therapies for ischemic stroke. Curr Treat Options Cardiovasc Med 2016;18:29.

56. Puy L, De Guio F, Godin O, Duering M, Dichgans M, Chabriat $H_{1}$ et al. Cerebral microbleeds and the risk of incident ischemic stroke in CADASIL (Cerebral Autosomal Dominant Arteriopathy With Subcortical Infarcts and Leukoencephalopathy). Stroke 2017;48:2699-2703.

57. Lee JS, Ko K, Oh JH, Park JH, Lee HK, Floriolli D, et al. Cerebral microbleeds, hypertension, and intracerebral hemorrhage in cerebral autosomal-dominant arteriopathy with subcortical infarcts and leukoencephalopathy. Front Neurol 2017;8:203.

58. Rinnoci V, Nannucci S, Valenti R, Donnini I, Bianchi S, Pescini

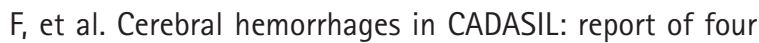
cases and a brief review. J Neurol Sci 2013;330:45-51.

59. Petty GW, Brown RD Jr, Whisnant JP, Sicks JD, O'Fallon WM, Wiebers DO. Ischemic stroke subtypes: a population-based study of functional outcome, survival, and recurrence. Stroke
2000;31:1062-1068.

60. SPS3 Investigators, Benavente OR, Hart RG, McClure LA, Szychowski JM, Coffey CS, et al. Effects of clopidogrel added to aspirin in patients with recent lacunar stroke. $N$ Engl J Med 2012;367:817-825.

61. Hanger HC, Wilkinson TJ, Fayez-Iskander N, Sainsbury R. The risk of recurrent stroke after intracerebral haemorrhage. $J$ Neurol Neurosurg Psychiatry 2007;78:836-840.

62. Haley KE, Greenberg SM, Gurol ME. Cerebral microbleeds and macrobleeds: should they influence our recommendations for antithrombotic therapies? Curr Cardiol Rep 2013;15:425.

63. Gurol ME. Nonpharmacological management of atrial fibrillation in patients at high intracranial hemorrhage risk. Stroke 2018;49:247-254.

64. Kwok CS, Shoamanesh A, Copley HC, Myint PK, Loke YK, Benavente OR. Efficacy of antiplatelet therapy in secondary prevention following lacunar stroke: pooled analysis of randomized trials. Stroke 2015;46:1014-1023.

65. Diener HC, Bogousslavsky J, Brass LM, Cimminiello C, Csiba L, Kaste $M$, et al. Aspirin and clopidogrel compared with clopidogrel alone after recent ischaemic stroke or transient ischaemic attack in high-risk patients (MATCH): randomised, double-blind, placebo-controlled trial. Lancet 2004;364:331337.

66. Shinohara Y, Katayama Y, Uchiyama S, Yamaguchi T, Handa $\mathrm{S}$, Matsuoka $\mathrm{K}$, et al. Cilostazol for prevention of secondary stroke (CSPS 2): an aspirin-controlled, double-blind, randomized non-inferiority trial. Lancet Neurol 2010;9:959-968.

67. Culebras A, Rotta-Escalante R, Vila J, Domínguez R, Abiusi G, Famulari $A$, et al. Triflusal vs aspirin for prevention of cerebral infarction: a randomized stroke study. Neurology 2004; 62:1073-1080.

68. Uchiyama S, Shinohara Y, Katayama Y, Yamaguchi T, Handa $\mathrm{S}$, Matsuoka $\mathrm{K}$, et al. Benefit of cilostazol in patients with high risk of bleeding: subanalysis of cilostazol stroke prevention study 2. Cerebrovasc Dis 2014;37:296-303.

69. Kim BJ, Lee EJ, Kwon SU, Park JH, Kim YJ, Hong KS, et al. Prevention of cardiovascular events in Asian patients with ischaemic stroke at high risk of cerebral haemorrhage (PICASSO): a multicentre, randomised controlled trial. Lancet Neurol 2018;17:509-518.

70. Kim JS, Kwon SU, Uchiyama S. Cilostazol research in Asia: can it be applied to European and American patients? Int J Stroke 2015;10 Suppl 1:1-9.

71. Mohr JP, Thompson JL, Lazar RM, Levin B, Sacco RL, Furie $K L$, et al. A comparison of warfarin and aspirin for the prevention of recurrent ischemic stroke. N Engl J Med 2001;345:14441451. 
72. The Stroke Prevention in Reversible Ischemia Trial (SPIRIT) Study Group. A randomized trial of anticoagulants versus aspirin after cerebral ischemia of presumed arterial origin. Ann Neurol 1997;42:857-865.

73. Bath PM, Wardlaw JM. Pharmacological treatment and prevention of cerebral small vessel disease: a review of potential interventions. Int J Stroke 2015;10:469-478.

74. Wilson D, Al-Shahi Salman R, Klijn CJ, Lip GY, Werring DJ. Intracerebral haemorrhage, atrial fibrillation, and anticoagulation. Lancet 2015;386:1736-1737.

75. Wilson $D$, Jäger HR, Werring DJ. Anticoagulation for atrial fibrillation in patients with cerebral microbleeds. Curr Atheroscler Rep 2015;17:47.

76. Wang Z, Soo YO, Mok VC. Cerebral microbleeds: is antithrombotic therapy safe to administer? Stroke 2014;45:2811-2817.

77. Topcuoglu MA, Liu L, Kim DE, Gurol ME. Updates on prevention of cardioembolic strokes. J Stroke 2018;20:180-196.

78. Biffi $A$, Kuramatsu JB, Leasure $A$, Kamel $H$, Kourkoulis $C$, Schwab $K$, et al. Oral anticoagulation and functional outcome after intracerebral hemorrhage. Ann Neurol 2017; 82:755-765.

79. Lammie GA. Hypertensive cerebral small vessel disease and stroke. Brain Pathol 2002;12:358-370.

80. Wardlaw JM, Smith C, Dichgans M. Mechanisms of sporadic cerebral small vessel disease: insights from neuroimaging. Lancet Neurol 2013;12:483-497.

81. Arima $H_{1}$ Anderson $C$, Omae T, Liu L, Tzourio C, Woodward M, et al. Perindopril-based blood pressure lowering reduces major vascular events in Asian and Western participants with cerebrovascular disease: the PROGRESS trial. J Hypertens 2010;28:395-400.

82. Yusuf S, Diener HC, Sacco RL, Cotton D, Ounpuu S, Lawton $W A$, et al. Telmisartan to prevent recurrent stroke and cardiovascular events. N Engl J Med 2008;359:1225-1237.

83. SPS3 Study Group, Benavente OR, Coffey CS, Conwit R, Hart $R G, M c C l u r e ~ L A$, et al. Blood-pressure targets in patients with recent lacunar stroke: the SPS3 randomised trial. Lancet 2013;382:507-515.

84. Biffi A, Anderson CD, Battey TW, Ayres AM, Greenberg SM, Viswanathan $A$, et al. Association between blood pressure control and risk of recurrent intracerebral hemorrhage. JAMA 2015;314:904-912.

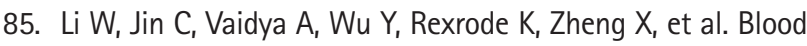
pressure trajectories and the risk of intracerebral hemorrhage and cerebral infarction: a prospective study. Hypertension 2017;70:508-514.

86. Huang Y, Cai X, Li Y, Su L, Mai W, Wang S, et al. Prehypertension and the risk of stroke: a meta-analysis. Neurology
2014;82:1153-1161.

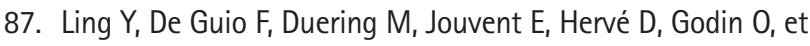
al. Predictors and clinical impact of incident lacunes in cerebral autosomal dominant arteriopathy with subcortical infarcts and leukoencephalopathy. Stroke 2017;48:283-289.

88. Pettersen JA, Keith J, Gao F, Spence JD, Black SE. CADASIL accelerated by acute hypotension: Arterial and venous contribution to leukoaraiosis. Neurology 2017;88:1077-1080.

89. Dekker JM, Girman C, Rhodes T, Nijpels G, Stehouwer CD, Bouter LM, et al. Metabolic syndrome and 10-year cardiovascular disease risk in the Hoorn Study. Circulation 2005; 112:666-673.

90. Najarian RM, Sullivan LM, Kannel WB, Wilson PW, D'Agostino RB, Wolf PA. Metabolic syndrome compared with type 2 diabetes mellitus as a risk factor for stroke: the Framingham Offspring Study. Arch Intern Med 2006;166:106-111.

91. Zhu S, McClure LA, Lau $H$, Romero JR, White $C L$, Babikian V, et al. Recurrent vascular events in lacunar stroke patients with metabolic syndrome and/or diabetes. Neurology 2015;85:935-941.

92. Boulanger M, Poon MT, Wild SH, Al-Shahi Salman R. Association between diabetes mellitus and the occurrence and outcome of intracerebral hemorrhage. Neurology 2016;87:870-878.

93. Palacio S, McClure LA, Benavente OR, Bazan C 3rd, Pergola P, Hart RG. Lacunar strokes in patients with diabetes mellitus: risk factors, infarct location, and prognosis: the secondary prevention of small subcortical strokes study. Stroke 2014;45:26892694.

94. Stroke Prevetion by Aggressive Reduction in Cholestrol Levels (SPARCL) Investigators, Karam JG, Loney-Hutchinson L, McFarlane SI. High-dose atorvastatin after stroke or transient ischemic attack: the Stroke Prevention by Aggressive Reduction in Cholesterol Levels (SPARCL) Investigators. J Cardiometab Syndr 2008;3:68-69.

95. Amarenco P, Bogousslavsky J, Callahan A 3rd, Goldstein LB,

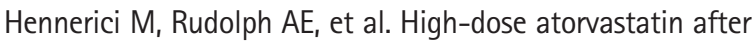
stroke or transient ischemic attack. N Engl J Med 2006; 355:549-559.

96. Goldstein LB, Amarenco P, Szarek M, Callahan A 3rd, Hennerici $M$, Sillesen $H$, et al. Hemorrhagic stroke in the stroke prevention by aggressive reduction in cholesterol levels study. Neurology 2008;70(24 Pt 2):2364-2370.

97. Hassan A, Hunt BJ, O'Sullivan M, Bell R, D'Souza R, Jeffery $S$, et al. Homocysteine is a risk factor for cerebral small vessel disease, acting via endothelial dysfunction. Brain 2004; 127(Pt 1):212-219.

98. Maron BA, Loscalzo J. The treatment of hyperhomocysteinemia. Annu Rev Med 2009;60:39-54. 
99. Cavalieri M, Schmidt R, Chen $C$, Mok V, de Freitas GR, Song $S$, et al. $B$ vitamins and magnetic resonance imaging-detected ischemic brain lesions in patients with recent transient ischemic attack or stroke: the VITAmins TO Prevent Stroke (VITATOPS) MRI-substudy. Stroke 2012;43:3266-3270.

100. Ikram MA, Vernooij MW, Hofman A, Niessen WJ, van der
Lugt A, Breteler MM. Kidney function is related to cerebral small vessel disease. Stroke 2008;39:55-61.

101. Greenberg SM, Rosand J, Schneider AT, Creed Pettigrew L, Gandy SE, Rovner B, et al. A phase 2 study of tramiprosate for cerebral amyloid angiopathy. Alzheimer Dis Assoc Disord 2006;20:269-274. 